\title{
Archives
}

\section{L'apport de François Beaudin : la mise en place du rapport Deschênes}

\section{DIANE BAILLARGEON}

Volume 46, numéro 2, 2017

$50^{\mathrm{e}}$ anniversaire de la DGDA

URI : https://id.erudit.org/iderudit/1040377ar

DOI : https://doi.org/10.7202/1040377ar

Aller au sommaire du numéro

\section{Éditeur(s)}

Association des archivistes du Québec (AAQ)

ISSN

0044-9423 (imprimé)

2369-9256 (numérique)

Découvrir la revue

Citer cet article

Baillargeon, D. (2017). L’apport de François Beaudin : la mise en place du rapport Deschênes. Archives, 46(2), 15-26. https://doi.org/10.7202/1040377ar
Résumé de l'article

Ce texte présente les années charnières du Service des archives de l'Université de Montréal, débutant lorsque François Beaudin arrive à la direction du service en avril 1972. Il hérite alors d'une situation décriée par son prédécesseur, Luc-André Biron, dans un mémoire déposé à la Commission conjointe du Conseil de l'Université et de l'Assemblée universitaire. Le rapport de la Commission, préparé par le juge Deschênes, en accepte les principales recommandations donnant un nouveau souffle au Service. S'appuyant sur ces recommandations, François Beaudin transformera le Service des archives en profondeur durant son court mandat de quatre ans : création de la Commission des archives, embauche de personnel qualifié qui feront leur marque sur la scène archivistique, approbation des premières règles de conservation, mise en place de dépôt de documents semi-actifs, etc.
Ce document est protégé par la loi sur le droit d'auteur. L'utilisation des services d'Érudit (y compris la reproduction) est assujettie à sa politique d'utilisation que vous pouvez consulter en ligne.

https://apropos.erudit.org/fr/usagers/politique-dutilisation/ 


\section{ANNIVERSAIRE DE LA DGDA}

\section{L'apport de François Beaudin: la mise en place du rapport Deschênes}

\section{DIANE BAILLARGEON}

Directrice, Division de la gestion de documents

et des archives, Université de Montréal

\section{INTRODUCTION}

\section{Mais qui donc était François Beaudin?}

Le deuxième directeur du Service des archives de l'Université de Montréal n'est pas un néophyte. Successivement licencié de théologie, archiviste de l'archevêché de Montréal et de la ville de Québec, membre fondateur de l'Association des archivistes du Québec (AAQ), formé aux méthodes du Records management par sa fréquentation assidue des congrès de la Society of American Archivists, chargé de cours en archivistique à l'Université Laval, à I'Université d'Ottawa ainsi qu'à I'Université du Québec à Montréal, François Beaudin est nommé Archiviste de I'Université, le 4 avril 1972. II quittera son poste en 1976 pour devenir le Conservateur des Archives nationales du Québec, avant d'être nommé sous-ministre adjoint responsable du patrimoine, des archives et des musées puis président de la Commission de toponymie du 
Québec durant 7 ans. C'est donc peu de dire qu'il avait fait ses preuves et qu'on lui reconnaissait de grandes qualités de gestionnaire et d'archiviste.

Résumons son passage par un seul mot: transformation. En quatre ans, il a réussi à transformer en profondeur le Service des archives pour lui donner, à peu de choses près, les contours qu'on lui connaît encore aujourd'hui.

\section{1. ÉTAT DES LIEUX DU SERVICE DES ARCHIVES À SON ARRIVÉE}

Pour bien comprendre à quel point le mandat de François Beaudin a été déterminant pour le devenir du Service des archives, il convient de faire un pas en arrière pour présenter l'état des lieux au moment de son arrivée en poste et les actions posées par son prédécesseur, le premier archiviste universitaire.

Lorsqu'il prend la tête du Service des archives, celui-ci compte 7 personnes, y compris l'Archiviste, et occupe des locaux peu adaptés aux besoins du service, situés dans le pavillon de l'administration. Dans le rapport annuel de 1970-1971, le premier directeur, Luc-André Biron écrit:

Le Dépôt actuel a maintenant atteint un point de saturation tel qu'il est dorénavant impossible de faire l'acquisition d'un seul classeur et qu'il faudra résoudre un problème aigu d'espace au cours de l'année qui vient. Cette exiguïté empêche aussi l'engagement d'un personnel supplétif. (Biron, 1971, p. 1)

Nous pourrions donc croire que le service stagne et que l'avenir est sombre pour lui. Et pourtant, nous sommes à l'aube de grands bouleversements et tout est en place pour récolter les fruits qu'a semés Luc-André Biron dans son mémoire déposé à la Commission conjointe du Conseil de l'Université et de l'assemblée universitaire'.

Profitant de ce grand chantier qui cherche à moderniser l'ensemble de I'Université, I'archiviste Luc-André Biron y dépose, le 27 mai 1969, un mémoire qui marquera à jamais le cours de l'histoire du Service des archives. II y déclare d'entrée de jeu:

Parmi les services d'établissements de toute nature, il en est un qui, pour méconnu qu'il soit parfois, n'en demeure 
pas moins précieux et rentable: c'est le Service des archives. C'est en effet grâce aux nombreux documents conservés au Dépôt [...] que la vie administrative de l'établissement maintient la garantie de son institution, la sécurité de sa constitution, le sillage de son histoire. [...] Le but de ce mémoire est de provoquer, tant du côté de l'Administration de l'Université de Montréal que du côté de ses facultés, une véritable prise de conscience de la nécessité d'organiser une collaboration constructive pour faire face au problème, sans cesse grandissant, de: LA GESTION DES ARCHIVES. (Université de Montréal, 1969, p. 1-2)

La première partie de son mémoire de vingt-quatre pages pose un diagnostic de la situation qui prévaut. Véritable cours d'introduction à l'archivistique pour les non-initiés que sont les membres de la Commission, Luc-André Biron y définit ce que sont les archives et explique qu'elles se «forment » dans les bureaux au fil des activités quotidiennes des centaines de personnes qui y œuvrent. Il distingue les « archives de direction» créées dans les hautes sphères de la hiérarchie administrative, importantes, mais peu abondantes, des "archives d'exécution» produites à tous les échelons de la hiérarchie, "le niveau inférieur produisant les archives les plus volumineuses et de l'intérêt durable le plus limité. » (Université de Montréal, 1969, p. 5) II passe ensuite en revue les différents concepts de "plan de classement», il compare les coûts de conservation dans les bureaux versus ceux dans un dépôt de préarchivage et il milite en faveur d'un programme de conservation des archives dont les principaux objectifs seraient de:

- préserver et protéger les documents vitaux et essentiels;

- supprimer les duplicata;

- établir pour chaque série de documents une période de conservation;

- réduire l'achat de classeurs;

- étudier les possibilités d'emploi du microfilm;

- construire ou aménager un dépôt de préarchivage. (Université de Montréal, 1969, p. 9)

La deuxième partie lui sert à définir un programme de gestion des archives qui repose sur les concepts les plus avancés en matière de gestion documentaire, ceux qui sont à la base de ce qui s'enseigne et se 
pratique encore aujourd'hui, à quelques adaptations technologiques près. Le mémoire se termine par la présentation d'un plan d'action en douze étapes qui va de la création des ARCHIVES CENTRALES de I'Université de Montréal au contrôle de l'achat des classeurs à l'instauration d'une

Commission d'archives [...] qui serait présidée par le Secrétaire général de I'Université [...] qui recommanderait toutes les mesures nécessaires au fonctionnement efficace des divers fonds d'archives, déciderait de l'orientation des nombreux dépôts dans l'Université, des modalités de leur développement ou de leur amélioration, adopterait les règlements généraux des fonds d'archives et, sur consultation des Conseils des facultés, les règlements particuliers aux archives précieuses ou confidentielles. (Université de Montréal, 1969, p. 20-21)

À la fin de l'année 1969, la Commission fait siens plusieurs des éléments du programme proposé par Luc-André Biron, et recommande:

147. Que soit créé le service des archives centrales de l'Université, sous la responsabilité du secrétaire général de I'Université et sous la direction immédiate de l'archiviste de l'Université.

148. Que ce service soit chargé de recueillir et conserver, suivant les méthodes les plus modernes, tous les documents d'intérêt public de l'Université.

149. Que ce service soit également chargé d'aider à uniformiser graduellement dans l'Université la procédure de classification et d'archivage des dossiers.

150. Que soit créé un dépôt central des archives, chargé de constituer et de tenir à jour un fichier général de toutes les archives de l'Université, de ses facultés, écoles et services.

151. Que soit créé un dépôt de préarchivage pour la conservation immédiate des documents des facultés, écoles et services qui ont cessé d'être d'utilisé courante.

152. Que soit constituée une commission des archives de I'Université. 
153. Que cette commission soit présidée [...] par le secrétaire général et qu'elle comprenne, outre l'archiviste de l'Université, un doyen, un professeur, un étudiant et un membre du personnel nommés par le Comité exécutif après consultation de l'archiviste.

154. Que cette commission puisse, par des règlements soumis à l'approbation préalable du Comité exécutif, décider des documents à déposer aux archives centrales ou au dépôt de préarchivage, de la procédure de classification et d'archivage que devront suivre les facultés, écoles et services et, en général, de tout ce qui sera nécessaire au fonctionnement efficace du service des archives centrales.

155. Que l'archiviste de l'Université soit chargé de voir à la mise à exécution, sous la responsabilité du secrétaire général, des règlements adoptés par la commission des archives. (Université de Montréal, Commission conjointe du Conseil et de l'Assemblée universitaire, 1970, p. 159)

Posant un regard plein d'espoir sur les retombées positives que ne manqueront pas d'avoir ces décisions sur l'avenir du service et de I'Université tout entière, Luc-André Biron écrit, dans le rapport annuel du service pour l'année 1970-1971:

Ces décisions administratives ont à la fois novatrices et hardies eu égard à ce qui se fait dans la plupart des universités actuelles. Elles sont, notamment, bien en avance par rapport au type archaïque de dépôts d'archives que I'on fait dépendre des bibliothèques universitaires. Elles impliquent l'intervention directe de l'archiviste au stade de l'organisation des papiers dans les facultés, écoles et services. [...] II est sans doute difficile, à une époque où les pratiques de l'austérité sont particulièrement impératives, de songer à mettre en marche un système de "gestion des archives courantes» à l'amplitude de l'Université. Cependant, telle est l'unique solution pour doter notre institution d'un instrument indispensable grâce auquel I'ADMINISTRATION - et en finalité I'HISTOIRE - de I'Université seront au mieux servies. II n'est pas de rôle plus noble que de faire I'histoire plutôt que de l'écrire. (Biron, 1971, p. 2 et 7) 


\section{LA MISE EN PLACE DES RECOMMANDATIONS DU RAPPORT DESCHÊNES.}

C'est donc dire qu'à son arrivée à la tête du Service des archives en avril 1972, la voie est toute tracée pour François Beaudin. Mais si le programme est bien défini, tout ou presque, reste à faire. II y a bien le programme de microfilmage des documents essentiels qui a été instauré dès 1968, ce qui a permis de «photographier » «tous les registres des grands corps universitaires [...] de même que tous les documents vitaux de l'Université [et de poursuivre le] microfilmage des registres des procès-verbaux des conseils des facultés de manière normale et régulière. » (Biron, 1971, p. 4) Cependant, la réalisation des principales recommandations est encore à venir, dont la création de la Commission des archives et l'adoption des premiers règlements qui viendront façonner le programme de gestion des archives.

François Beaudin sait bien que pour mettre en place toutes ces recommandations, il faut du personnel compétent. Luc-André Biron avait déjà indiqué que, sur les six personnes qui composaient le personnel du Service des archives, mis à part le directeur, seules deux personnes avaient déjà de telles qualifications, mesdames Madeleine Roy, documentaliste, et Suzanne Lalonde, son adjointe. Le plus urgent était donc de rehausser le niveau de compétence du personnel. C'est ce à quoi il s'attelle dès son arrivée en poste.

\section{PREMIÈRES TRANSFORMATIONS}

\subsection{Les ressources humaines}

Dès l'année 1972-1973, les effectifs passent de sept à neuf personnes par l'embauche d'un adjoint à l'archiviste, Carol Couture, et d'un responsable des archives historiques, Jacques Ducharme. Retenez bien ces noms, ils feront leur marque dans l'archivistique québécoise. De plus, un poste de technicien responsable de la documentation imprimée est créé alors que trois employés voient leurs postes être réévalués. Lorsqu'il quitte le Service des archives en septembre 1976, le personnel compte dix postes permanents à temps plein, grâce à l'embauche de deux personnes à la section du microfilm, sans compter l'embauche de personnel temporaire à temps plein ou partiel. 
Mais alors qu'il y avait, en 1972, 6 employés de bureau, en plus de l'Archiviste, aujourd'hui on y trouve un autre cadre (I'adjoint à l'archiviste), un professionnel (le responsable des archives historiques), quatre techniciens en documentation, un technicien en microphotographie, une aide technique, et une secrétaire. (Université de Montréal, Secrétariat général, 1976, p. i)

Pour réussir à accomplir la tâche colossale à laquelle il s'est attelé, François Beaudin accueille aussi de nombreux stagiaires. Ainsi, en 19731974, ce n'est pas moins de vingt-six personnes qui feront un stage dans les différentes sections du service, stages variant de quelques heures à soixante-dix jours. Durant cette seule année, quarante-quatre personnes auront ainsi œuvré au Service des archives.

La formation du personnel et la diffusion de la connaissance constituent déjà une préoccupation pour le nouvel Archiviste universitaire. Ainsi les employés du Service des archives assistent ou participent au congrès de l'AAQ qui se tient, en 1974, à I'Université de Montréal. De plus, le technicien de la section du microfilm est envoyé suivre une formation de deux semaines au Service central du microfilm des Archives publiques du Canada, aujourd'hui, Bibliothèque et Archives Canada. Les liens se tissent avec le milieu de l'enseignement, et «l'Archiviste, I'adjoint à l'Archiviste et le Responsable des archives historique et le Technicien en microfilm [donnent] des cours d'archivistique à Québec et à Montréal. » (Beaudin, 1974, p. 5)

Ce double souci de la formation continue du personnel du Service et de la transmission des connaissances et du savoir-faire archivistique sera une des grandes caractéristiques du service. Pensons à l'ouvrage Les archives au XX siècle. Une réponse aux besoins de l'administration et de la recherche, de Carol Couture et Jean-Yves Rousseau², publié par le Secrétariat général de l'Université de Montréal en 1982, qui fait en quelque sorte la recension des méthodes de travail adoptées par le Service des archives de I'Université de Montréal. Le même désir de partager son savoir amène Denys Chouinard et Michel Champagne, respectivement responsable de la section des archives historiques et archiviste, à publier, en 1987, Le traitement d'un fonds d'archives: ses documents historiques, qui sert encore d'ouvrage de référence dans l'enseignement du traitement définitif d'un fonds d'archives.

Sous la gouverne de François Beaudin, le Service des Archives aura aussi le souci de documenter ses procédures de travail. Ainsi, soixante 
« méthodes» seront rédigées afin de s'assurer qu'une information exacte et complète puisse être mise à la disposition de tous ceux qui œuvrent au service. Avec le nombre impressionnant de stagiaires et de personnel temporaire, à temps plein ou partiel qui travaillent au Service des archives durant une année, la rédaction de ces procédures est un élément essentiel de la rigueur et de la constance du travail à réaliser.

\subsection{Les locaux et équipements}

Ayant recruté des archivistes qualifiés pour le seconder, François Beaudin peut s'attaquer à l'éternel problème des espaces. Le Service des archives quitte le pavillon de l'administration pour le pavillon principal et aménage un dépôt de préarchivage au $21^{\mathrm{e}}$ étage de la tour qui sera progressivement agrandi pour en occuper six étages complets.

La section du microfilm prend de l'ampleur, lorsque François Beaudin quitte la direction en septembre 1976, la section peut compter sur quatre caméras, dont trois ont été récupérées d'autres unités de I'Université, ce qui permet d'augmenter la production de microfilms à des fins de sécurité ou de substitution. Cette section fonctionnera jusqu'en 2008 alors que l'atelier de microfilm se convertit à la numérisation sérielle de masse avec toujours le même souci de protéger et de donner accès aux documents vitaux de l'Université.

Le Service commence aussi à s'informatiser et, en 1976, 22 fichiers informatiques totalisant 72000 informations sont à la disposition du personnel, dont la mécanisation de l'inventaire des dossiers inactifs du personnel administratif et du personnel enseignant.

\subsection{Publications et relations publiques}

Le Service publie ses premiers instruments de recherche, dont le Guide de consultation à l'intention des chercheurs et le Cadre de classement des archives de l'Université de Montréal. Durant les quatre années du mandat de François Beaudin, le Service produit vingt-cinq publications dont certaines seront rééditées à plusieurs reprises et seront vendues aussi loin qu'en Finlande.

Fort de son nouveau mandat qu'il convient de faire connaître, le Service des archives confie au Centre de l'audiovisuel le soin de réaliser 
un « diaporama » sur le service. Tous les moyens sont bons pour atteindre ses objectifs.

\section{MISE EN PLACE DE LA COMMISSION DES ARCHIVES}

C'est en juin 1972, soit quelque mois à peine après I'arrivée de François Beaudin à la tête du Service des archives, que la Commission des archives tient sa première réunion. C'est cependant à partir d'octobre que les travaux débutent véritablement. Les premiers membres en seront, outre le président et secrétaire général Jacques Girard et l'archiviste François Beaudin qui agit à titre de secrétaire, Jean-Guy Benoist, directeur du Centre informatique de gestion, Roland Rivest, vice-doyen de la Faculté des arts et des sciences, Claude Touchette, professeur de la Faculté des sciences de l'éducation et Michel Landry, représentant des étudiants.

Durant les premières années de son existence, elle tient plusieurs réunions par année, car le travail à abattre est considérable. Premièrement, édicter les Règlements qui encadreront la gestion des archives à l'Université. Pour accélérer les choses et éviter que le rejet d'une section d'un Règlement retarde l'adoption des autres parties, la Commission décide de voter plusieurs règlements successifs. En 1973, les quatre premiers règlements sont ainsi adoptés. Le premier règlement stipule que: "Font partie des archives de l'Université, tous les documents qui appartiennent à l'Université ou dont celle-ci est le dépositaire » (Université de Montréal, 1973), le deuxième enjoint aux unités de verser au Service des archives, tous documents antérieurs à 1967, le troisième constitue une délégation de pouvoir du Comité exécutif à la Commission des archives pour tout ce qui concerne l'élimination des documents et le quatrième traite de l'accessibilité des archives.

\section{L'OPÉRATION TRANSFERT}

Le deuxième règlement, adopté par le Comité exécutif le 15 mars 1973, enjoignait à toutes les unités académiques et administratives de I'Université de transférer les documents postérieurs à 1967 au Service des archives. En trois courts articles, la Commission des archives venait de lancer une plus vaste opération de rapatriement de documents semiactifs. Appelée "opération transfert» I'application de ce Règlement mobilise des ressources encore aujourd'hui, car certains des documents 
reçus à l'époque, sans règles de conservation, sont encore à être traités en 2016.

Pour mener à bien cette opération d'envergure, il faut bien se préparer: concevoir des formulaires de transfert, diffuser l'information, solliciter les unités qui n'ont pas toutes le même désir de se soumettre au règlement, les soutenir dans la réalisation de cette opération, recevoir, inventorier et localiser tous ces documents. C'est un ambitieux, mais nécessaire travail qui permet de rapatrier plus de mille pieds linéaires de documents durant la seule année 1973.

\section{CALENDRIER DE CONSERVATION ET SYSTÈME OFFICIEL DE CLASSIFICATION}

Une des plus grandes réalisations de l'ère Beaudin sera la mise en place du calendrier de conservation et du système officiel de classification (SOC). Dix ans avant l'adoption de la Loi sur les archives qui viendra répandre l'utilisation de cet outil d'évaluation et de gestion des archives, les dispositions réglementaires de I'Université de Montréal permettent à la Commission des archives de statuer sur l'élimination des documents et d'approuver les règles de conservation. Dans son rapport annuel 1975-1976, qui sert aussi de bilan de son administration, François Beaudin écrit: "L'Université est maintenant dotée d'un ensemble de politiques concernant ses archives, suite aux travaux de la Commission des archives. En particulier, elle possède un bon embryon de calendrier de conservation. » (Université de Montréal, Secrétariat général, 1976, p. iii)

Durant la seule année 1975-1976, quarante-neuf nouvelles règles ont été adoptées, dont trente-huit pour la seule Direction des finances, ce qui permet l'élimination de l'équivalent de 115 tiroirs de classeurs et de 48 boîtes de documents. Qui plus est, ces règles de conservation ont mené au microfilmage de 30 tiroirs de classeurs de documents qui ont ensuite été éliminés.

C'est aussi durant l'administration de François Beaudin qu'est mis en place un comité ad hoc, formé de sept personnes, qui a pour mandat:

1. d'étudier la mise sur pied d'un système de classement uniforme des archives à l'Université de Montréal ;

2. d'étudier des systèmes existant dans d'autres institutions aux États-Unis, au Canada ou ailleurs; 
3. d'établir des contacts avec le plus grand nombre possible de personnes œuvrant à tous les niveaux à l'Université de Montréal pour prévoir les avantages et les problèmes que pourrait poser l'adoption d'un classement uniforme;

4. de faire rapport à la Commission des archives et lui soumettre les solutions qu'il propose ainsi que les modes d'application de ces solutions. (Université de Montréal, Secrétariat général, 1976, p. 8)

Les travaux de ce comité aboutiront à l'adoption du Système officiel de classification (SOC) qui sera en vigueur à l'Université jusqu'en 2006.

\section{CONCLUSION}

Le bilan que I'on peut tirer des années 1972-1976 durant lesquelles François Beaudin a dirigé le Service des archives est plus que positif. Si Luc-André Biron en a été le laboureur, précurseur des changements à venir en déposant un mémoire fort remarqué à la Commission conjointe du conseil et de l'assemblée universitaire, Beaudin en a été celui qui a veillé à ce que les recommandations ne tombent pas dans I'oubli, qu'elles soient mises en application avec diligence et ingéniosité.

II a su obtenir les ressources nécessaires pour réaliser ce plan d'action ambitieux. Basé sur les méthodes du Record management anglo-saxon, sans oublier l'archivistique de tradition plus européenne, Beaudin a insufflé au Service un dynamisme, une rigueur et un désir de partager l'information qui ne s'est jamais démentis.

Son autre grand talent a été de s'entourer de professionnels qui feront ensuite leur marque sur la scène nationale et internationale et qui ont fait du Service des archives de I'Université de Montréal, un service modèle dont toute institution peut s'enorgueillir.

II n'est aussi que justice de rendre hommage à ceux qui l'ont appuyé dans son travail, les

recteurs Roger Gaudry et Paul Lacoste, la direction éclairée fournie par les secrétaires généraux Jacques Girard et Juliette Barcelo, leurs adjoints Nicole Trudeau-Bérard et Michel Lespérance et la collaboration assidue et empressée de [son] personnel [...] en particulier de [son] adjoint Carol Couture, 
[...] sans oublier le précieux apport de tous ceux qui ont été membres de la Commission des archives. (Université de Montréal, Secrétariat général, 1976, p. v)

\section{NOTES}

1. Mieux connue sous le nom de la Commission Deschênes, la Commission conjointe du Conseil de l'Université et de l'Assemblée universitaire est créée en 1968 et tient sa première réunion le 21 novembre 1968 sous la présidence du juge Jules Deschênes. Elle a pour mandat d'étudier le rôle de l'Université dans la société, le rôle de ses composantes et les relations entre les composantes de l'Université. Le rapport de la Commission a donné lieu à d'importantes modifications de l'organisation de l'Université.

2. Jean-Yves Rousseau est embauché à titre d'adjoint à l'Archiviste au moment où Carol Couture devient le directeur du Service des archives.

\section{BIBLIOGRAPHIE}

BEAUDIN, F. (1974). Rapport annuel 1973-1974. Montréal.

BIRON, L.-A. (1971). Rapport annuel 1970-1971. Montréal.

UNIVERSITÉ DE MONTRÉAL. (1969). Les archives de I'Université de Montréal: Mémoire présenté par Luc-André Biron, Archiviste, à la Commission conjointe du Conseil et de l'assemblée universitaire, le 27 mai 1969. Montréal.

UNIVERSITÉ DE MONTRÉAL. (1973). Définition des archives de I'Université de Montréal, Règlement no 1 adopté par le Comité exécutif. Montréal.

UNIVERSITÉ DE MONTRÉAL. COMMISSION CONJOINTE DU CONSEIL ET DE L'ASSEMBLÉE UNIVERSITAIRE. (1969). L'Université, son rôle, le rôle de ses composantes, les relations entre ses composantes. Montréal: Presses de l'Université de Montréal.

UNIVERSITÉ DE MONTRÉAL. SECRÉTARIAT GÉNÉRAL. (1976). Le Service des archives de l'Université de Montréal: Rapport annuel pour l'année universitaire 1975-1976 par François Beaudin Archiviste de l'Université. Montréal. 